\title{
Construire des géographies: formes et savoirs émergents de la géographie Introduction au cahier thématique
}

\section{Jean-Bernard Racine, Lausanne}

Ce dernier numéro de l'année 2008 établit un lien entre, d'une part, une pratique géographique qui s'est voulue moderne au niveau de ses référents conceptuels et théoriques, de ses outils quantitatifs ou qualitatifs et de ses objets d'étude, et d'autre part les cheminements déjà tracés par une nouvelle génération de jeunes géographes. Accrochés à de nouveaux rêves géographiques, ces chercheurs ont choisi des sujets de recherche dans lesquels on peut supposer qu'entrent aujourd'hui tout autant les préoccupations des géographes que celles des citoyens. Cette dynamique a été bien illustrée par les différents auteurs, rencontrés par le soussigné dans le cours de sa carrière et ayant accepté de contribuer à l'ouvrage d'hommage recensé en fin de ce volume et réalisé sous la direction d'ANTOnio Da Cunha et de Laurent Matthey (2007). D'une certaine manière ce numéro s'en veut déjà le prolongement.

La thématique renvoie de fait à deux axes de réflexion complémentaires à ce qui a été offert dans l'ouvrage collectif autour de la question des nouveaux objets du savoir géographique: un axe lié aux objets émergents et un axe lié aux manières les plus efficaces aujourd'hui d'en traiter. Antonio Da Cunha et LaUrent Matthey ouvrent le débat en développant la problématique de l'ouvrage qu'ils ont dirigé au travers de ses plus intéressantes intuitions. Respectivement professeur et chargé de recherche à l'Institut de Géographie de l'Université de Lausanne (IGUL), ils témoignent par leur propos d'une évolution sensible qui doit autant, semble-t-il, à l'évolution de la contextualité qu'à l'enrichissement des outils disponibles au double plan de la réflexion théorique et de l'action.

Les propositions du professeur ChrIsTIAN VANDERMOTTEN permettent de revisiter les rapports entretenus entre la science et la société. L'auteur évoque plus particulièrement ce qui est sans doute le problème central de la géographie et qui concerne les liens entre les paradigmes mobilisés dans la science géographique d'une part et la position sociale des géographes d'autre part. De manière peut-être inattendue au départ, le texte rencontre très évidemment l'objet de ce numéro, ne serait-ce qu'en légitimant du point de vue historique et logique le rapprochement entre géographie sociale et géographie physique, contre tant d'idées devenues courantes ces vingt dernières années. Ce savoir ré-émergent est incarné dans «une 〈simple et hétérodoxe science sociale géohistorique〉 (TAYLOR 1996, cité dans VANDERMOTTEN, ce cahier, page 234) transcendant les corporatismes disciplinaires».

Maître d'enseignement et de recherche à l'IGUL, d'abord formé en économie politique puis en démographie, Christophe MAger engage ses lecteurs sur une autre voie, peut-être complémentaire. Sa contribution cherche à identifier, au long d'une sorte de cheminement de petits cailloux blancs, ce que sa discipline, qu'il qualifie encore simplement de «géographie économique», peut tirer de la confrontation entre Nouvelle Sociologie Économique et Nouvelle Géographie Économique. Est-ce l'occasion d'un nième plaidoyer pour un plus grand irrespect des frontières disciplinaires? Sans doute, mais cette fois en s'appuyant sur une critique rigoureuse des contenus conceptuels et des postulats dont ils dérivent, montrant que par-delà les oppositions apparentes, elles-mêmes liées à des représentations distinctes de l'économie et des sociétés, la complémentarité analytique des approches est tout à fait indispensable. L'auteur montre alors que les deux démarches évoquées explorent des aspects complémentaires de l'économie capitaliste dans laquelle baigne la réalité géographique, «la spatialité historicisée des économies». On retiendra avec lui que «l'analyse géographique est propice à cette inscription des réflexions à la croisée de différents champs du savoir».

Professeure à l'IGUL, responsable de l'enseignement de la cartographie, Micheline Cosinschi met le doigt sur une véritable découverte, paradoxalement inscrite dans la mise en évidence d'un invariant discursif: le modèle ternaire. Ce modèle, inscrit dans la logique naturelle, est présenté avec la même rigueur que lorsque l'auteur défendait la scientificité du discours géographique au cours de la période dite du «quantitatif» - dont on ne dira jamais assez à quel point la représentation pouvait être abusive. Encore une fois, et dans un domaine peu fréquenté par les épistémologues et pourtant consubstantiellement lié à la pratique de la géographie, on se plaira à reconnaître dans ce travail non seulement un «savoir émergent» mais peut-être beaucoup plus. Entre les concepts d'ordre, de hiérarchie et d'organisation, telle que celle-ci est irréductible tout en étant engendrée par les deux premiers, «configuration représentative d'états de choses possibles» (EveræRT-Desmedt 1990, cité dans CosinschI, ce 
cahier, page 248), prolongée par la triade information, signification, communication, ne découvre-t-on pas la logique même de tout discours géographique? Cela est une manière de «sortir de l'obscurité conceptuelle qu'entretient la complexité» et, à propos de la carte, médiatrice et révélateur du décodage des rapports de l'homme au territoire, de montrer l'origine de pratiquement tous les concepts utilisés, dont on voit bien qu'ils permettent de mieux viser l'interprétation d'une réalité première: la ville.

Viennent enfin deux articles consacrés à ce qui est, depuis longtemps, réalité statistique, expérience quotidienne, la réalité première de toute géographie humaine. Bien qu'on le sache depuis plus d'un demisiècle, cette réalité s'est transformée de manière radicale depuis les premières critiques de l'exode rural puis des formes de l'expansion métropolitaine, de dilution de l'espace urbain, d'interrogation sur la fin des villes et sur l'émergence des cités lisières. L'heure est pour beaucoup au retour à la ville et à la réinvention des valeurs de l'urbanité, singulièrement observable en Amérique du Nord, d'ailleurs en mal de rêve urbain européen. La Suisse commence à enregistrer le phénomène.

Géographie-urbaniste, maître-assistante à l'IGUL, participante de l'Observatoire universitaire du développement durable, BÉATRICE BoChET évoque une préoccupation jointe à un état d'âme. Il s'agit de l'amour des villes qui, loin des tristesses de la ville mal-aimée plaide simultanément - toujours la même préoccupation convergente entre une réflexion nouvelle sur les objets et les savoirs émergents - pour l'inscription à l'agenda des géographes d'un champ de recherche associant leurs préoccupations tant géographiques que citoyennes. Elle approfondit et donne une première illustration du contenu possible d'une idée force depuis longtemps défendue par le soussigné, tout au long de ses quelque quarante ans d'enseignement et de recherche en géographie urbaine: le fait qu'une bonne appréhension de la réalité urbaine, la saisie de la présence cognitive de l'espace urbain, suppose la mise en perspective réciproque de plusieurs dimensions, mor- phologique, socio-démographique et structuro-fonctionnelle, mais également le registre socio-culturel et socio-affectif tel qu'il est vécu par ses habitants. En d'autres termes, la nécessité pour le géographe d'apprendre à saisir les affects urbains et à mobiliser le registre émotion et la manière dont les émotions sont socialement partagées au quotidien de la vie urbaine. La manière dont l'auteur propose d'y greffer la notion de prise affective, telle que développée par ailleurs par Augustin Berque (Berque, A. (1990): Médiance. De milieux en paysages. - Montpellier: GIP Reclus), est totalement originale. Elle mérite évidemment qu'on en tente l'expérimentation, encore à venir, en se demandant en outre dans quelle mesure l'expérience sensible peut conditionner l'expression discursive.

Plus factuel, le dernier article rappelle enfin, tout en la nuançant quant à son impact sur différents types de populations, l'importance nouvelle que prennent ou re-prendront les centres urbains comme mode d'occupation privilégié du territoire. Dans le cadre d'un projet de recherche du FondS NATIONAL SUISSE DE LA RECHERCHE SCIENTIFIQUE (FNS) consacré au «Retour en ville», les professeurs neuchâtelois Etienne Piguet et Ola SöDERström ainsi que les jeunes chercheurs Patrick Rérat et Roger Besson montrent qu'une réurbanisation est peut-être en cours, invitant bien évidemment les géographes à la réflexion et à l'action.

C'est pourquoi, au chapitre des comptes-rendus, le lecteur de ce numéro trouvera une note d'ensemble sur les plus récents travaux de la géographie urbaine française, les uns et les autres choisis comme représentant tous, à propos de la ville et de l'urbain à l'aube d'un nouveau siècle, une géographie au cœur de l'ensemble des sciences humaines. Ces analyses devraient permettre d'introduire l'effort de synthèse entre les différents courants de recherche qui s'imposent actuellement.

Prof. Dr. Jean-Bernard Racine, Décanat GSE, Amphipôle 211, Université de Lausanne, CH-1015 Lausanne, Suisse.

e-mail: Jean-Bernard.Racine@unil.ch 